\title{
Estrategias y Estilos de Enseñanza en la Clase Magistral de estudios oficiales de Danza Española y Flamenco \\ Teaching Strategies and Styles in the Master Class of Official Spanish Dance and Flamenco Studies \\ *Rosa de las Heras Fernández,** María Espada \\ *Universidad Internacional de la Rioja (España), **Universidad Politécnica de Madrid (España)
}

Resumen. Los estudios formales de danza se han llevado a cabo en España en los Conservatorios, estableciéndose actualmente tres niveles de enseñanza: Elemental, Profesional y Superior. Es allí donde se llevan a cabo diversas Clases Magistrales impartidas por distintos profesionales y donde el alumno recibe técnicas innovadoras de enseñanza, pues el Estilo de Enseñanza predominante en estos centros suele ser el tradicional. En este estudio se investiga la percepción y satisfacción de 89 alumnos de enseñanzas Profesionales y Superiores en una Clase Magistral. Se utilizó una metodología mixta, con técnicas de triangulación. Los resultados muestran gran satisfacción del alumnado siendo mayor en los estudiantes de enseñanzas Superiores.

Palabras clave: educación artística, danza, educación superior, educación profesional.

Abstract. Formal dance studies have been carried out in Spanish Conservatories, currently establishing three levels of teaching: Elementary, Professional, and Superior. It is there where various Master Classes are held by different professionals and where the students receive innovative teaching techniques, as the predominant Teaching Style in these centers is usually the traditional one. This study investigates the perception and satisfaction of 89 students of Professional and Superior teachings in a Master Class. A mixed methodology was used, with triangulation techniques. The results show great satisfaction of the students, being it greater in the students of Superior teachings. Keywords: artistic education, dance, higher education, professional teachings.

\section{Introducción}

La enseñanza en los Conservatorios de Danza en España

Los estudios formales de Danza en España, se han llevado a cabo fundamentalmente en los Conservatorios. Estas enseñanzas pasan a denominarse enseñanzas Elementales, Profesionales y Superiores según la Ley Orgánica 2/2006, de 3 de mayo, de Educación (p.45-47). En ella se situaba la Danza Española como especialidad. El baile Flamenco pasó a ser otra de las especialidades de las enseñanzas Profesionales de Danza, de seis años de duración, en virtud del Real Decreto 85/2007 (p.6250). Tres años más tarde, cuando se publica el Real Decreto 632/2010, se regula el contenido básico de las enseñanzas artísticas Superiores de Grado en Danza, incluyendo el estilo de baile: Danza Española y Flamenco. Los estudios Profesionales suelen finalizarse a la edad de 18 años y no dan obtención a titulación oficial y los estudios Superiores están destinados a profundizar en aspectos de la interpretación y partes teóricas y ofrecen títulos equivalentes a Grado. En la formación en estos centros se imparten Clases Magistrales de diferentes profesionales expertos en las distintas materias y disciplinas. Estudios como los de Creech (2009) nos muestran la percepción de los estudiantes frente a estas clases Magistrales impartidas en Conservatorios de Música y como dichas clases pueden ofrecer ideas frescas para abordar la interpretación. No obstante, apenas existe literatura científica sobre la percepción y satisfacción de los estudiantes en las Clases Magistrales llevadas a cabo en Conservatorios de Danza, a pesar de que estas clases pueden ofrecer nuevas metodologías y estrategias didácticas que ayuden a motivar al alumnado. En esta línea,

Fecha recepción: 18-02-20. Fecha de aceptación: 23-04-20

Rosa de las Heras Fernández

rosa.heras@unir.net autores como Ryan y Deci (2000) concluyen que la satisfacción que genera una actividad constituye un indicador del comportamiento y de la implicación de las personas en dicha actividad, de forma que a mayor satisfacción, más motivación y mejor logro de los objetivos que promueve la actividad. Por su parte, Junco (2010) explica que la motivación ayuda al alumnado a ser decisivo en actividades, puesto que tiene que asumir una posición ante una situación nueva y esto hay que tenerlo en cuenta en el proceso de aprendizaje. Sin embargo, el clima motivacional del aula no sólo depende de los estudiantes, la intervención docente es la base para que los alumnos den sentido al proceso de enseñanza y aprendizaje. Es por ello, que cada vez más la comunidad científica centra su atención en los métodos de enseñanza utilizados por el profesorado, así como la satisfacción y percepción del alumnado hacia estos nuevos métodos, en el caso que nos ocupa: los Conservatorios de Danza. Así, encontramos estudios que muestran los estilos educativos en la formación dancística en las escuelas profesionales de danza (Ferreiro-Pérez, 2015).

\section{Los Estilos de Enseñanza}

Los estilos de enseñanza son herramientas que el profesorado posee para poder proporcionar una amplia gama de situaciones de enseñanza- aprendizaje a sus alumnos, permitiendo así el máximo desarrollo del proceso (Mosston y Ashworth, 2008). El espectro de estilos de enseñanza creado por Muska Mosston, es el modelo por antonomasia utilizado en el ámbito de la educación física (Espada, Fernández \& Calero, 2019). Por ello, los estilos de enseñanza son una herramienta metodológica importante para llevar a cabo las clases de Educación Física (Fernández y Espada, 2017).

La actual versión del espectro de Mosston recoge 11 estilos, que se estructuran en dos grandes grupos: reproductivos y productivos. En los primeros (estilos A o comando, B o enseñanza basada en la tarea, C o enseñanza recíproca, D o autoevaluación y E o estilos de inclusión) el 
protagonismo se centra en el profesor, y los alumnos principalmente reproducen las tareas. En el grupo de los estilos productivos (F o descu-brimiento guiado, $\mathrm{G}$ o resolución de problemas, $\mathrm{H}$ o divergente, I o para alumnos iniciados, J o programa individualizado y $\mathrm{K}$ o autoenseñanza) los estudiantes tienen un mayor control de la tarea y se les invita al descubrimiento y la creatividad para la resolución de las diferentes actividades (Mosston y Ashworth, 2008).

Si analizamos los estilos de enseñanza que se suele llevar a cabo en los Conservatorios de España, tiende a utilizarse un enfoque tradicional. Tal y como expone Robledo (2003) esta metodología se caracteriza por ser «tradicional, academicista, memorístico, orientado a la adquisición de habilidades, destrezas en los aspectos técnicos-interpretativos» (p. 66). Por lo tanto, actualmente en danza se emplean estilos que se circunscriben en el grupo de estilos reproductivos de la clasificación del espectro de Mosston.

Más concretamente, el estilo de enseñanza más utilizado actualmente en la enseñanza de la Danza, es el denominado Comando, el cual se caracteriza por el total protagonismo del profesor en la toma de decisiones. El alumnado ejecuta, sigue un modelo y obedece (Mosston y Asworth, 1993). Sin embargo, sería interesante plantearse la posibilidad de utilizar otros estilos de enseñanza, concretamente del grupo de los estilos productivos, para la enseñanza de la Danza en los Conservatorios. Uno de estos estilos podría ser el denominado Estilo Divergente, en este caso, existe más de una respuesta correcta siempre que se encuentren dentro de los parámetros de la situación (Espada, Fernández \& Calero, 2019). Es el profesor quien plantea un problema que los alumnos tendrán que resolver (Mosston y Asworth, 1993). De esta forma, se dota al alumnado de mayor autonomía y libertad en la toma de decisiones, lo que ayudaría a aumentar la motivación del alumnado (Conde y Almagro, 2013). En el ámbito de la Danza el Estilo Divergente se puede llevar a cabo a través de trabajos de improvisación, en los que el alumno tras recibir la información inicial por parte de su profesor proponga diversas respuestas.

\section{Estrategia en la práctica de la enseñanza}

La estrategia en la práctica de la enseñanza es la forma particular de abordar los diferentes ejercicios que componen la progresión de enseñanza de una determinada habilidad motriz (Delgado, 1991). La estrategia que sigue el docente a la hora de abordar la tarea también puede facilitar la obtención de destrezas motrices y cognitivas. Así, las estrategias de práctica siguen alguna de las vías del proceso de pensamiento como son las síntesis y el análisis. Tradicionalmente se han conocido en la Didáctica como el método analítico y el método sintético o global (Delgado, 1991).

En esta línea, en los Conservatorios de Danza, se suele utilizar una estrategia de carácter global, en la que el alumnado repite la secuencia rítmica en su totalidad, como ocurre en la práctica del zapateado. Sin embargo, la comprensión de las diferentes partes de la tarea a través de la estrategia analítica permite al alumnado un aprendizaje más significativo. No obstante, tal y como se ha expuesto anteriormente, las metodologías de enseñanza en el Danza Española y el Flamenco, apenas utilizan el estilo de enseñanza divergente y cuando utilizan la estrategia analítica en el comando, no ofrecen siempre una comprensión de los contenidos, pues por ejemplo, cuando un profesor de danza, para enseñar un zapateado, divide los pasos, no es capaz de explicar los ritmos que se encuentran en ellos, pues se enseña de oído (De las Heras-Fernández, 2017) que es la manera en la que ellos a su vez han aprendido (Syrmpas \& Digelidis, 2014). La forma de estudio consiste en la memorización de fragmentos y su continua repetición hasta que son capaces de interpretar la secuencia al completo (Casas-Mas, Pozo \& Montero, 2014). Así, recursos y estrategias didácticas para desarrollar el conocimiento, memorización, improvisación y creación del ritmo a través del movimiento corporal, empleadas en metodologías musicales como Dalcroze (Juntunen \& Hyvönen, 2004), permanecen ajenas a estas enseñanzas. «Crear» forma parte de un pensamiento superior según la taxonomía de Bloom (Krathwohl, 2002). Según esta taxonomía, en el proceso de aprendizaje, la dimensión cognitiva conduce desde el recuerdo, pasando por la comprensión, la siguiente aplicación, el análisis y la evaluación, hasta la creación. Sin la comprensión del ritmo del zapateado, presentes en la Danza Española y en el Flamenco, no podríamos llegar a la dimensión cognitiva del aprendizaje. El alumno en la enseñanza tradicional adquiere comprensión corporal de los movimientos del zapateado, sin embargo, carecería de conocimiento cognitivo del ritmo que realiza al zapatear pues como hemos dicho antes, aprenden el ritmo solamente «de oído» (De las Heras-Fernández, 2017). Por otra parte, para el desarrollo de la creatividad en música, (que también está presente en la práctica del ritmo del zapateado), Lago (2006), establece cuatro períodos: imitación, improvisación, experimentación y creación, sin embargo y a pesar de que en el baile flamenco la improvisación y la creatividad están presentes en la interpretación (Gamboa y Núñez, 2017), apenas existen estudios sobre su enseñanza. En cualquier caso, el proceso suele iniciarse desde la observación y la imitación para alcanzar, más adelante, la expresión individual, propia y única (Peñalver, 2019). Llegados a este punto y siguiendo la taxonomía de Bloom en el que «crear» forma parte de un pensamiento de mayor orden, cabría preguntarse si sería conveniente aplicar otros estilos de enseñanza y estrategias, utilizados en otros ámbitos, tales como la Educación Física, junto con metodologías para la enseñanza del ritmo usadas en Educación Musical. Así, partiendo del estilo de enseñanza Comando, (imitación) y la estrategia analítica (comprensión) llegar posteriormente al estilo divergente (improvisación) como forma de acercarse a la creatividad. La imitación de los movimientos y ritmos del zapateado, la comprensión de esos ritmos, que se llevaría a cabo mediante metodologías musicales para el aprendizaje de contenidos rítmicos, como figuras y células rítmicas presentes en la práctica del zapateado y la práctica improvisada de los ritmos que previamente han comprendido. Así se procura que estilos, estrategias y metodologías ayuden a la mejora del conocimiento y comprensión en la práctica del zapateado en los estilos de Danza Española y Flamenco.

\section{Objetivos}

En este estudio se investiga la percepción y satisfacción de los estudiantes de Conservatorio en una Clase Magistral de zapateado. El propósito ha sido doble, puesto que por un 
lado se ha pretendido (a) examinar la percepción de los estudiantes en el estilo de enseñanza comando con estrategia analítica y estilo divergente, con los contenidos y recursos planteados y (b) conocer si existen diferencias entre los estudiantes del Conservatorio de Danza Profesional y Superior en relación a la metodología empleada así como la motivación en la clase de zapateado.

\section{Metodología}

La investigación siguió una metodología mixta con técnicas de triangulación, utilizándose un cuestionario AMPET, de motivación de logro para el aprendizaje de Educación Física Nishida (1984; 1988; 1989 y 1991) y una batería de preguntas con respuesta dicotómica y abiertas (Paramo, 2018).

En la parte cualitativa, el diseño y validación de las preguntas se llevó a cabo a través del juicio de expertos, construida a través de diferentes categorías bibliográficas y que serviría para llevar a término los objetivos de investigación propuestos. De esta manera se construye la información, la misma realidad mostrada de diferente manera que permite al investigador disponer de mayor «evidencia» sobre las afirmaciones que pretende sostener (Scribano \& Sena, 2009).

La parte cuantitativa es de corte descriptivo y transversal (Alvira, 2002). En el desarrollo de la metodología, los procedimientos que se siguieron fueron los propios de la encuesta (Cea, 2004).

Todos los sujetos participaron de forma anónima y voluntaria en el estudio, teniendo en cuenta todos los procedimientos éticos aplicables en la recopilación y tratamiento de los datos. La investigación fue aprobada por la secretaría de estudios de los Centros donde se realizó la intervención. La profesora contaba con 38 años de experiencia en danza, 20 de ellos como interprete, docente profesional y una amplia formación y experiencia en Educación Musical.

\section{Muestra}

Se realizó un muestreo no probabilístico y por conveniencia, debido a las limitaciones para acceder a una población tan exclusiva. La muestra total es de 89 individuos entre los que el 48,3\% eran estudiantes y profesores del Grado Profesional y el 51,7\% eran del Grado Superior de Danza Española y Flamenco. En el Grado Profesional un total de $93 \%$ de mujeres y $7 \%$ de varones y con una edad entre 16 y 55 años $(22.98 \pm 10.365)$ y en el Grado Superior el $87 \%$ de mujeres y el $13 \%$ de varones con una edad comprendida entre los 19 y 39 años (23.49 \pm 4.159$)$. En cuanto a la experiencia artística en Teatros, Tablaos, Peñas y otros espacios escénicos, el Grado Profesional cuenta con esta experiencia en un $58,1 \%$ mientras el $41,9 \%$ no cuenta con experiencia artística. En el Grado Superior la experiencia artística es mayor, llegando al 78,3\% y no cuenta con dicha experiencia un $19,6 \%$ de los encuestados. En cuanto a la experiencia docente el $39,5 \%$ de estudiantes y profesores del Grado Profesional habían ejercido docencia en ámbitos formales y no formales (Heras-Monastero, 2013) y el 58,1\% no habían impartido docencia. En el Grado Superior la experiencia docente es también más alta, con un 76,1\%, en enseñanzas no formales, mientras que un $23,9 \%$ no ha ejercido docencia.

\section{Instrumento}

Para registrar la percepción y satisfacción de los estudiantes ante la Clase Magistral impartida se utilizaron dos instrumentos:

a) Una batería de preguntas, con respuestas dicotómicas y abiertas. La validez del instrumento se llevó a cabo a través de juicio de expertos (Alarcón, Aidé, Balderrama Trápaga \& Edel Navarro, 2017). La batería de preguntas al completo se puede visitar en el siguiente enlace web: https:/ /reunir.unir.net/bitstream/handle/123456789/8419/ Preguntas $\% 20 \% 20$ propuesta $\% 20$ didactica2.pdf? sequence $=1 \&$ isAllowed $=y$

b) El otro instrumento utilizado en el estudio fue traducido y validado en habla hispana para medir la motivación de logro para el aprendizaje de Educación Física Nishida (1984; 1988; 1989 y 1991). La adaptación Española (Ruiz-Pérez, Moreno-Murcia, Ramón-Otero y Alias-García, 2015), consta de 32 ítems y 4 dimensiones. Se puntúa en una escala tipo Likert de 1 (Totalmente en desacuerdo) a 5 (Totalmente de acuerdo). La consistencia interna del instrumento original fue elevada (.86) así como el coeficiente de correlación testrestest que fue de .87. Para el adecuado desarrollo del presente trabajo, se seleccionaron las dimensiones: competencia motriz autopercibida,( ítems 4, 8, 12, 19, 22, 29 y 30) Competencia motriz comparada (ítems $3,7,11,18$ y 25) Compromiso con el aprendizaje (ítems 2, 6, 19, 14, 17, 21, 24, 28 y 31) y Ansiedad y Agobio ante el fracaso (ítems 1, 5, 9, $13,16,20,23,27$ y 32$)$.

\section{Procedimiento}

Se impartieron tres Clases Magistrales, una en el Conservatorio Superior de Danza de Madrid y las otras dos en el Conservatorio Profesional de danza de Córdoba, tanto para alumnos como para profesores. La duración de las clases fue de 2 horas, 10 minutos. Antes de finalizar cada sesión, se procedió a repartir los cuestionarios y preguntas para su cumplimentación.

Durante el transcurso del trabajo de campo y a su término, se efectuaron las tareas de control y supervisión, se comprobó que los cuestionarios se hallaran total y adecuadamente cumplimentados, es decir, que se hubieran recogido todas las informaciones previstas y además verificando que los datos realmente hubieran sido recogidos como se debían recoger (Miquel et al., 2000).

\section{Análisis estadístico}

\section{La batería de preguntas}

El análisis de contenido de las respuestas se llevo a cabo inicialmente a través de la transcripción y codificación de las palabras clave a través del software ATLAS.TI, v. 8. 4. 2. El contenido se clasificó en función de las categorías obtenidas en la revisión bibliográfica previa y emparejamiento temático:

a) Categoría 1: Datos personales (Kvale, 2011). A partir de ella, se pretendió conocer aspectos relativos a los datos sociales, profesionales y académicos de los entrevistados.

b) Categoría 2: Metodología, estrategias y estilos de enseñanza (Mosston \& Ashworth, 2002). Los conocimientos adquiridos y la percepción de los estudiantes con respecto a las estrategias didácticas llevadas a cabo en la clase. 
Se generaron una lista de códigos y familias de códigos asignados a múltiples fragmentos además de mapas conceptuales para la construcción de la teoría (Varguillas, 2006). Así, el análisis de la información permitió la discriminación de las categorías y sub-categorías presentes en el Gráfico 1 (ver Figura 1).

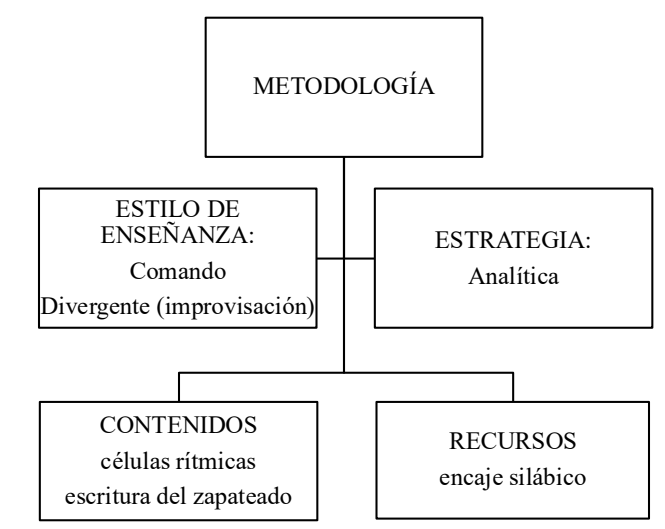

Figure 1. Árbol de categorías Atlas.ti.

\section{El AMPET}

Para realizar el análisis estadístico se recurrió al programa SPSS v.25.0 (SPSS Inc., E.E.U.U). Se utilizaron pruebas de estadística descriptiva e inferencial. En la estadística descriptiva, se hallaron datos estadísticos de frecuencia tales como: frecuencias, media y desviaciones típicas. En la estadística inferencial, para comprobar la normalidad de las distribuciones, se utilizó el test de Kolmogorov-Smirnov. Las variables que cumplían una distribución normal, por ello se utilizó la prueba $t$ de Student para muestras independientes con el fin de analizar la relación existente entre las variables. Asimismo, se utilizó el test de Levene para evaluar la homogeneidad de las varianzas, usando el criterio estadístico de significación de $p<.05$.

Finalmente, para identificar las correlaciones entre las diferentes dimensiones en cada uno de los grupos se utilizó el estadístico Pearson.

\section{Resultados}

\section{La batería de preguntas}

En la Tabla 1 se muestran la batería de preguntas en relación con la metodología empleada: la posible utilidad del conocimiento de las células rítmicas, su escritura, el recurso didáctico del encaje silábico, la posible aplicabilidad en la creación y la improvisación del ritmo del zapateado, la facilidad para la memorización y la percepción general sobre la propuesta didáctica. Así mismo, se señalan los porcentajes de satisfacción ante estos ítems en la enseñanza Profesional y Superior. Las respuestas con respecto a la utilidad de la escritura del zapateado, metodología, utilidad de la propuesta didáctica y recursos empleados en la Clase Magistral, resultaron positivas en ambos estudios, por encima del 74,4\% en los estudios Profesionales de Danza y $84,8 \%$ en el caso de los estudios Superiores. Sin embargo, en cuanto al aprendizaje de nuevos ritmos en la clase, el 73,9\% (Superior) y 83,7\% (enseñanza Profesional) afirma que no aprenden ritmos nuevos. Se señalan porcentajes positivos, aunque medianos en ambos estudios en lo referente a la pregunta sobre si la meto-
Tabla 1.

Bateria de preguntas, por porcentajes

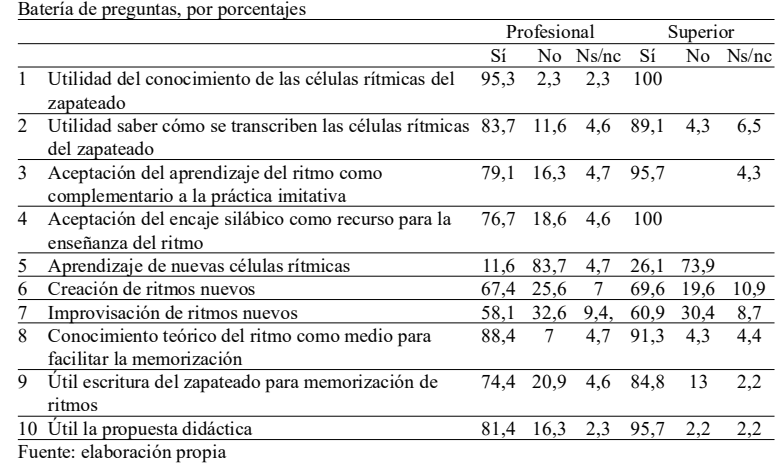

dología podría servir como medio para el desarrollo de la improvisación y la creación (entre el 58,1\% y el 69,6\%), siendo algo mayor en el caso de los Estudios Superiores

Las preguntas 2, 3 fueron de respuesta dicotómica y aluden a aspectos de contenido y metodológicos de la Clase. Las respuestas fueron mayoritariamente afirmativas: en el conocimiento de las células rítmicas $(83,7 \%$ enseñanzas Profesionales y $89,1 \%$ en el Superior) y en la transcripción del ritmo (79, $1 \%$ en la enseñanza Profesional y 95,7\% en la enseñanza Superior).

En la tabla 1, se muestran las respuestas de cada una de las preguntas formuladas, no obstante, los encuestados no siempre exponen los motivos de sus respuestas.

Con respecto al primer ítem, si consideran útil el aprendizaje de las células rítmicas,

los encuestados de estudios Profesionales y Superiores respondieron de forma afirmativa $(95,3 \%$ y $100 \%$ respectivamente). La respuesta común de mayor porcentaje en ambos estudios es «aporta conocimiento» (34,9\% en enseñanzas Profesionales y un $32 \%$ en enseñanzas Superiores). Otro factor que se indica en ambos planes de estudio es «facilitar en el aprendizaje» (14\% en enseñanza Profesionales) y la importancia en «facilitar la comprensión» ( $23 \%$ en el Superior). Otras justificaciones que se señalan con menores porcentajes en ambas enseñanzas son la de «mejorar la interpretación» (11,6 \% en el Profesional) y «la vinculación de la práctica del zapateado con el conocimiento rítmico». La «posibilidad de registro» es señalado únicamente en las enseñanzas Profesionales y «la facilidad para la creación» únicamente en los estudios Superiores.

La cuarta pregunta hace referencia a la utilidad del encaje silábico, en ambos estudios respondieron mayoritariamente de forma afirmativa $(76,7 \%$, enseñanzas Profesionales y $100 \%$ en estudios Superiores). Señalaron la «facilidad y claridad» (21,2\% en Profesionales y $21,7 \%$ en estudios Superiores), «mejor comprensión» (24,2\% estudios Profesionales y 19,6\% en Superiores) «la propia propuesta de encaje» (12,1\% en enseñanzas Profesionales y $13 \%$ en Superiores). Además de ello, otros aspectos indicados serían: «memorizar, notación, método didáctico y en enseñanzas elementales» $(24,3 \%$ en enseñanzas Profesionales y $21,5 \%$ en Superiores). El 18,6\% de los encuestados de enseñanzas Profesionales que respondieron de forma negativa, consideran que «es un impedimento la necesidad de conocimiento del Lenguaje Musical para la práctica del zapateado» (25\%) y que no lo consideran didáctico $(12,5 \%)$.

Con respecto a la quinta pregunta sobre si han aprendi- 
do nuevos ritmos. La mayoría admite no haber aprendido ritmos nuevos, sin embargo, los que afirman que sí (11,6\% en el Profesional y $6,1 \%$ en el Superior) indican «las negras, corcheas y semicorcheas» (40\% estudios Profesionales y $16,7 \%$ en Superiores) y «la figura rítmica del tresillo» $(20 \%$ enseñanzas Profesionales y 8,3\% en Superiores) siendo «la forma de nombrarlas» (20\% en estudios Profesionales y $25 \%$ en Superiores).

La sexta pregunta hace referencia a si a través de la metodología impartida consideran que se podría facilitar la creación de nuevos ritmos. Aunque la respuesta de ambos estudios es afirmativa, lo es de forma moderada $(67,4 \%$ en enseñanzas Profesionales y 69,6\% en estudios Superiores). La respuesta afirmativa común mayoritaria en ambos estudios es «mayor reflexión y comprensión» $(27,6 \%$ en enseñanzas Profesionales y $25 \%$ en Superiores). Otros factores que se señalan en las enseñanzas Profesionales son: «sistema de escritura del ritmo» $(24,1 \%)$, «una mejor forma de combinar ritmos» $(17,2 \%)$ y una «mayor facilidad» $(13,8 \%$. Otros comentarios comunes en ambos estudios aluden a «otras formas de experimentar», «aportar conocimiento». En las enseñanzas Superiores se indica «la importancia de esta metodología en enseñanzas elementales». Los que responden de forma negativa (25,6\% en enseñanzas Profesionales y $19,6 \%$ en Profesionales) la justificación común expuesta es que «no es necesaria la comprensión» $(36,4 \%$ en la enseñanza Profesional y 66,6\% en el Superior). Los alumnos de enseñanza Superior señalan además que «puede facilitar el aprendizaje pero no lo creación» $(11,1 \%)$.

En el análisis de las encuestas la improvisación y creación están desvinculadas, y no existen criterios comunes a la hora de entender que ambas se puede aprender y desarrollar. «No se desarrollan ni trabajan las capacidades que el profesional necesita para bailar en flamenco más tradicional fundamentado en la improvisación y la creación» (Arranz, 1998, p. 31). Probablemente sea porque en el flamenco el acto de improvisar se asocia únicamente al acto espontáneo y racial (Ríos-Ruiz, 2002) y por lo tanto, carente de comprensión racional.

La séptima pregunta pretende saber si la metodología podría favorecer la improvisación de ritmos nuevos. Al igual que ocurría en la sexta pregunta, aunque la respuesta de ambos estudios es afirmativa lo es de forma moderada (58,1\% en enseñanzas Profesionales y 60,9\% en estudios Superiores). Una de las respuestas comunes es «la vinculación del pensamiento rítmico al movimiento en la práctica del zapateado» (39,2\% en enseñanza Superiores y $12 \%$ en Profesionales). También se señalan en ambos estudios «los ejercicios planteados» ( $36 \%$ en enseñanzas Profesionales). También se indica en las enseñanzas Profesionales de manera particular el desarrollo de la creatividad (12\%) y el sistema de escritura del zapateado (8\%). Los alumnos de los estudios Superiores señalan «el desarrollo auditivo». Con respecto a las respuestas negativas (32,6 en enseñanzas Profesionales y 30,4\% en Superiores), la justificación común es «la no necesidad de comprensión y dificultad de la misma» (42,9\% Profesional y $35,7 \%$ en Superior) y otro factor señalado es «la dificultad de la escritura» (7,1\% en Profesional).

El ítem 8 pretende saber si el Conocimiento teórico del ritmo podría ser un medio para facilitar la memorización de los zapateados. La mayoría percibe que el conocimiento teórico podría facilitar la memorización $(88,4 \%$ en enseñanzas Profesionales y 91,3\% en Superiores). Los que respondieron afirmativamente en ambos grados coinciden en aspectos metodológicos impartidos como «la escritura y su vinculación con el encaje silábico» (31,6\% enseñanzas Profesionales y $35,7 \%$ en estudios Superiores) y «la comprensión» (21,1\% en enseñanzas Profesionales y $11,9 \%$ en Superiores), otros items señalados son «la simplicidad y rapidez» (13,2\% en enseñanzas Profesionales y 9,5\% en Superiores). Los que responden de forma negativa ( $7 \%$ en enseñanzas Profesionales y $4,3 \%$ en Superiores), reconocen principalmente que es debido a la falta de costumbre ( $100 \%$ enseñanzas Superiores y 33,3\% en Profesionales). Otro aspecto que se señala en las enseñanzas Profesionales es que «sería apropiado únicamente para enseñanzas elementales» $(33,3 \%)$.

En lo que respecta al noveno ítem si consideran útil la escritura del ritmo del zapateado, la mayoría percibe útil la escritura del ritmo del zapateado (74,4\% Profesional y $84,4 \%$ en el superior). Las razones comunes que se exponen en ambos estudios destacan que «el sistema de escritura se puede entender de forma clara y por lo tanto recordar y conservar fácilmente» (71,9\% en enseñanzas Profesionales y $64,1 \%$ en Superiores), otros aspectos que se señalan son «la escritura como herramienta de apoyo» (3,1\% en enseñanzas Profesionales y 2,6\% en Superiores). Otras justificaciones planteadas en los alumnos de enseñanzas Superiores (18,1\%) son: «sencillez, rapidez, aplicabilidad, sólo para ritmos sencillos, una forma de transcender, algo posible a largo plazo». Una minoría contesta de forma negativa ( $20,9 \%$ en enseñanzas Profesionales y un 13\% en Superiores) uno de los motivos que muestran en ambos estudios son «dificultades debido a necesitar la lectura del ritmo» (22,2\% en enseñanzas Profesionales y 33,4\% en enseñanzas Superiores). En este sentido, como recurso preferido, en las enseñanzas Profesionales se señala «el método tradicional a través de la transmisión auditiva, de oído» (22,2\%) y en el caso de alumnos de Superior la preferencia es «el uso del material audiovisual, como es el uso de las grabadoras de audio-vídeo» $(33,4 \%)$.

La última y décima pregunta hace referencia a si consideran útil la propuesta didáctica. Las respuestas fueron afirmativas en su mayoría (81,4\% en estudios Profesionales y 95,7\%). «El sistema de escritura del zapateado» es uno de los aspectos señalados en ambos estudios (100\% en enseñanzas Profesionales y 9,1\% en Superiores). Los alumnos de enseñanzas Superiores además señalan distintos aspectos en la utilidad de la propuesta didáctica $(45,5 \%)$, como: «la aplicabilidad y la ciencia, la facilidad en ordenar las ideas, el conocimiento y valor teórico que aporta, la metodología, la rapidez, eficacia y claridad, un recurso para el aprendizaje del flamenco y lo divertido del método».

\section{EI cuestionario AMPET}

En la prueba de Levene o prueba de homogeneidad de varianzas, se señalan las diferencias en las medias en cuanto las dimensiones percepción de la competencia motriz autopercibida, la competencia motriz comparada, compromiso con el aprendizaje y ansiedad y agobio ante el fracaso en los Grados Profesionales y Superiores de Danza Española y Flamenco (tabla 2). Por último, se procedió a realizar la 
Tabla 2

Medias Grado Profesional-Superior

\begin{tabular}{llcccc}
\hline & & $\mathrm{N}$ & Media & Desv. Tip & Error tip. de media \\
\hline Percepción competencia motriz profesional & 31 & 3,61 &, 72 &, 13 \\
\cline { 2 - 6 } autopercibida & superior & 37 & 3,34 &, 60 &, 09 \\
Percepción competencia motriz & profesional & 32 & 1,57 &, 44 &, 07 \\
\cline { 2 - 6 } comparada & superior & 39 & 1,38 &, 38 &, 06 \\
\hline Compromiso al aprendizaje & profesional & 32 & 4,36 &, 48 &, 08 \\
\cline { 2 - 6 } & superior & 38 & 4,43 &, 36 &, 05 \\
\hline Ansiedad y agobio al fracaso & profesional & 34 & 2,39 &, 89 &, 15 \\
\cline { 2 - 6 } & superior & 40 & 2,85 &, 80 &, 12 \\
\hline Fuente: Elaboración propia & & & & &
\end{tabular}

Prueba t para muestras independientes, en la cual se halló que existen diferencias estadísticamente significativas en la dimensión ansiedad y agobio ante el fracaso (Tabla 3) siendo superior en los estudiantes de estudios Superiores de Danza Española y Flamenco.

Tabla

Prueba de Levene para igualdad de varianzas

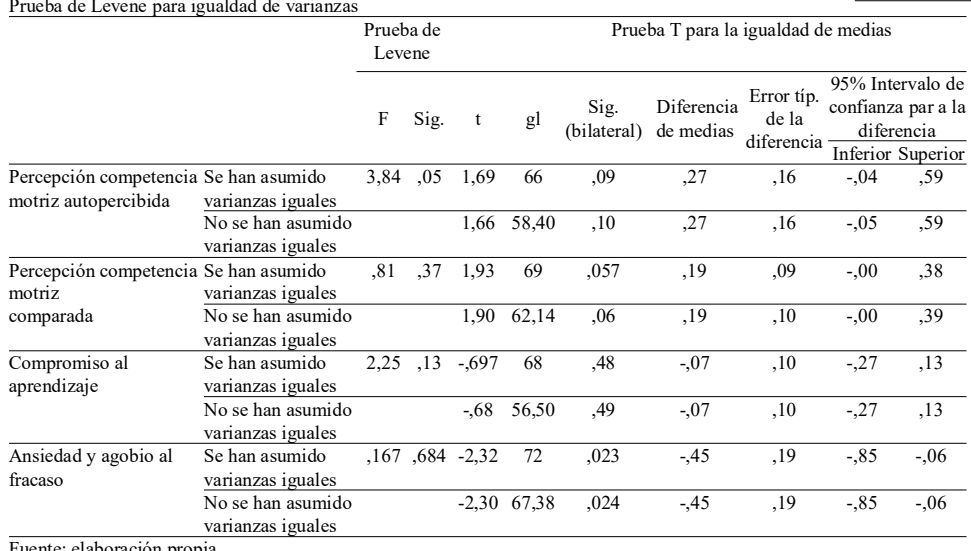

En la tabla 4 se muestra los resultados de estudiantes y profesores en el Grado Profesional en relación a las dimensiones: Competencia motriz autopercibida, Competencia motriz comparada, Compromiso con el aprendizaje y Ansiedad y Agobio ante el fracaso. Se puede comprobar que existen correlaciones positivas y altamente significativas en lo referente a la competencia motriz autopercibida y la competencia motriz comparada $(\mathrm{r}=.892 ; \mathrm{p}=.00)$. Además, se muestran correlaciones medias positivas en cuanto a la competencia motriz percibida y el compromiso al aprendizaje $(\mathrm{r}=$. $487 ; p=.00$ ). La competencia motriz autopercibida se relaciona negativamente con la ansiedad agobio al fracaso $(\mathrm{r}=-.475$; $\mathrm{p}=.00)$.

El compromiso al aprendizaje se correlaciona significativamente con la percepción de la competencia motriz comparada $(\mathrm{r}=.368 ; \mathrm{p}=.00)$ y ésta se relaciona negativamente con la ansiedad y agobio al fracaso $(r=-.346 \mathrm{p}=.04)$.

Tabla 4 .

Correlaciones dimensiones Grado Profesional

Correlaciones

\begin{tabular}{|c|c|c|c|c|}
\hline \multicolumn{5}{|l|}{$\begin{array}{c}\text { Correlaciones Grado Profesional } \\
\end{array}$} \\
\hline & (1) & (2) & (3) & (4) \\
\hline (1) Percepción competencia motriz & 1 & $892 * *$ &, $487 * *$ &,$- 475^{* *}$ \\
\hline \multirow[t]{2}{*}{ autopercibida } & & ,000 &, 005 & ,005 \\
\hline & 34 & 31 & 31 & 33 \\
\hline \multirow[t]{3}{*}{ (2) Percepción competencia motriz comparada } &, $892 * *$ & 1 & $368^{*}$ &,$- 346^{*}$ \\
\hline &, 000 & &, 038 & 045 \\
\hline & 31 & 35 & 32 & 34 \\
\hline \multirow[t]{3}{*}{ (3) Compromiso aprendizaje } &, $487^{* *}$ & ,368* & 1 &,- 293 \\
\hline &, 005 & ,038 & & 087 \\
\hline & 31 & 32 & 35 & 35 \\
\hline \multirow{3}{*}{ (4) Ansiedad Agobio al fracaso } &,$- 475^{* *}$ &,$- 346^{*}$ &,- 293 & 1 \\
\hline & ,005 & ,045 & ,087 & \\
\hline & 33 & 34 & 35 & 37 \\
\hline
\end{tabular}

En los alumnos del Grado Superior, (tabla 5) se puede comprobar que existen correlaciones medias positivas en lo referente a la competencia motriz autopercibida y la competencia motriz comparada $(r=.502 ; \mathrm{p}=.00)$, y correlaciona nega- tivamente con la ansiedad y agobio al fracaso $(\mathrm{r}=-.570 ; \mathrm{p}=.01)$. Existen correlaciones medias negativas entre la competencia motriz comparada y la ansiedad y agobio al fracaso $(\mathrm{r}=-, 396$; $\mathrm{p}=.01)$.

Tabla 5 .

Correlaciones dimensiones Grado Superior Correlaciones Grado Profesional

\begin{tabular}{lcccc}
\hline & $(1)$ & $(2)$ & $(3)$ & $(4)$ \\
\hline (1) Percepción competencia motriz & 1 &, $502^{* *}$ &, 200 &,$- 570^{* *}$ \\
autopercibida & 37 &, 002 &, 250 &, 000 \\
& 36 & 35 & 37 \\
\hline (2) Percepción competencia motriz comparada &, $502^{* *}$ & 1 &, 021 &,$- 396^{*}$ \\
&, 002 & &, 901 &, 013 \\
& 36 & 39 & 37 & 39 \\
\hline (3) Compromiso aprendizaje &, 200 &, 021 & 1 &,- 367 \\
&, 250 &, 901 & &, 023 \\
& 35 & 37 & 38 & 38 \\
\hline \multirow{2}{*}{ (4) Ansiedad Agobio al fracaso } &,$- 570^{* *}$ &,$- 396^{*}$ &,- 367 & 1 \\
&, 000 &, 013 &, 023 & \\
**. La correlación es significativa a nivel 0,01 (bilateral) & 39 & 38 & 40 \\
\hline *. La correlación es significante al nivel 0,05 (bilateral) & & & \\
\hline
\end{tabular}

*. La correlación es significante al nivel 0,05 (bilateral)

\section{Discusión}

Los objetivos del presente estudio eran analizar la percepción y satisfacción de una Clase Magistral, (a) en el estilo de enseñanza comando con estrategia analítica y estilo divergente y (b) si existen diferencias en la motivación en la clase de zapateado entre los estudiantes y profesores del Conservatorio de Danza Profesional y Superior en función la metodología utilizada

En cuanto al primer objetivo, el análisis de los datos muestra cómo los estudiantes y profesores de enseñanzas Profesionales y estudiantes de Grado Superior en los estilos de Danza Española y Flamenco, muestran alta satisfacción ante la metodología empleada en la Clase Magistral. Esto coincide con otros estudios que indican que las Clases Magistrales ofrecían valiosas oportunidades de interpretación, ideas frescas para abordar la interpretación y la técnica musical y el acceso a una comunidad profesional de práctica (Creech, Gaunt, Hallam, \& Robertson, 2009). En este sentido, en el presente trabajo se ha utilizado una metodología innovadora a través del estilo de enseñanza divergente para la instrucción del zapateado. Este estilo, promueve la creatividad del alumno, para ello se ofrece una total libertad de expresión y pensamiento para la creación de nuevos movimientos, la improvisación, la innovación, etc... El objetivo de este método es favorecer la oportunidad de desarrollar y descubrir soluciones originales al problema o situación planteada (Mosston \& Ashworth, 2001). Por otra parte, la estrategia analítica empleada en la que el alumno zapatea cada uno de los ritmos que se han analizado y comprendido, coincide con otros estudios, los cuales señalan que los movimientos corporales pueden ser una forma de comunicar la comprensión musical (Jacquier \& Pereira, 2010). Esto iría vinculado a la utilización del cuerpo como forma de expresión musical, en este caso concreto, rítmica, tal y como señalan autores como Dalcroze (Juntunen \& Hyvönen, 2004). La improvisación se ha incluido como práctica en el estilo divergente. Así, esta improvisación se ha llevado a cabo a través de la propuesta individual de los alumnos en los que cada uno realizaba su propio ritmo con los pies, dentro de los trabajados en clase magistral, interpretados en el orden y forma convenida. Así el alumno expuso su composición rítmica con los pies de una 
forma imaginativa, espontánea, y original (Peñalver, 2019). La improvisación es una estrategia importante para el aprendizaje musical (Peñalver, 2013) también en el aspecto rítmico (con la percusión de pies) pues la improvisación podría ofrecer una ruta para crear un diálogo íntimo con el potencial creativo de los alumnos y las interrelaciones entre los diferentes aspectos del aprendizaje (Wright \& Kanellopoulos, 2010). Además, la improvisación está vinculada con el proceso creativo (Casas, 2007), al ser uno de los posibles procedimientos de la creatividad, ya que estimula, necesariamente la capacidad de examinar, seleccionar, probar, que a fin de cuentas no es otra cosa que crear en el momento (Peñalver, 2019). De esta manera, podríamos llegar a «crear», como pensamiento de orden superior según la taxonomía de Bloom (Krathwohl, 2002).

El recurso del encaje silábico empleado en la Clase Magistral para el aprendizaje del ritmo, es una herramienta utilizada por diferentes autores como Kodaly (Gérard, 1991) o Hemsy de Gaiza (Hemsy de Gainza, 2002). Así por medio de esta estrategia se puede llegar a mejorar aprendizajes cognitivos sobre conceptos musicales rítmicos como las figuras musicales y las células rítmicas, que se realizan en la práctica del zapateado y cuyos contenidos cognitivos también son abordados en otras investigaciones (Rusinek, 2004). Así, la práctica del zapateado, no se limitaría únicamente al ámbito psicomotriz como sucede en la enseñanza tradicional con el aprendizaje «de oído» (De las Heras-Fernández, 2017).

Finalmente en lo referente al segundo objetivo, los resultados muestran diferencias estadísticamente significativas en los diferentes planes de estudio. Los estudiantes de enseñanzas Superiores mostraron un mayor porcentaje de satisfacción a la metodología empleada y esto se relaciona por un lado, con otro estudio donde el alumnado de estilo flamenco en el Grado Superior de música valora favorablemente los aspectos que le resultan novedosos (Torres, OrtegaRuz, \& Hidalgo, 2017) y por otro, otro estudio que muestra que las concepciones sobre el aprendizaje que tienen los estudiantes tienden a ser más sofisticadas a medida que su edad y nivel educativo aumentan (Bautista, Pérez-Echeverría, Pozo \& Brizuela, 2012).

Respecto a las correlaciones existentes en las diferentes dimensiones en ambos grupos, enseñanzas Profesionales y Grado Superior, vemos que en los estudiantes de enseñanza Profesional las correlaciones son más altas y significativas se dan entre la competencia motriz autopercibida y la competencia motriz comparada. Además, estas dos variables correlacionan de forma media y significativa con el compromiso al aprendizaje. Igualmente, los alumnos del Grado Superior correlacionan de forma positiva y significativa la competencia motriz autopercibida y la competencia motriz comparada. Cabe destacar, que los estudiantes de ambas enseñanzas coinciden en que cuanto mayor sea la competencia motriz autopercibida y la competencia motriz comparada, menor será la ansiedad y el agobio ante el fracaso.

Como conclusión final cabría señalar que la implantación de metodologías, estilos de enseñanza, estrategias y recursos diferentes en los estudios Profesionales y Superiores de Danza Española y Flamenco podría ser beneficiosa en el aprendizaje y motivación del alumnado, al igual que otras propuestas educativas con carácter innovador (Canizales,
Ries \& Rodríguez, 2020) consiguiendo que el cambio de estrategias procedimentales fortalezca la comprensión y dimensión cognitiva, logrando mejores respuestas interpretativas y facilitando la creación.

\section{Referencias}

Alarcón, G., Aidé, L., Balderrama Trápaga, J.A., \& Edel Navarro, R. (2017), «Validez de contenido por juicio de expertos: propuesta de una herramienta virtual». Apertura (Guadalajara, Jal.), vol. 9, núm. 2, pp. 42-53.

Alvira, F. (2002), «Diseños de investigación social: Criterios operati- vos. En F. Alvira, M. García Ferrando \& J. Ibáñez (Comps.)». El análisis de la realidad social. Métodos y técnicas de investigación (3.a ed.) Madrid: Alianza editorial. pp. 99-125

Arranz,A. (1998), El baile flamenco, Madrid: Librerías deportivas Esteban Sanz, S.L.

Bautista, A., Pérez-Echeverría, M. P., Pozo, J. I., \& Brizuela, B. M. (2012), «Piano students' conceptions of learning, teaching, assessment, and evaluation». Estudios de psicología, vol. 33, núm. 1,pp.79-104.

Casas, P. (2007), «La improvisación en la metodología musical (de adentro hacia afuera)». El artista: revista de investigaciones en música y artes plásticas, vol. 4, pp.123-129.

Casas-Mas, A., Pozo, J.I., \& Montero, I. (2014). The influence of music learning cultures on the construction of teachinglearning conceptions. British Journal of Music-Education, vol. 31, núm. 3,pp. 319-342

Canizales, W., Ries, F., \& Rodríguez, C. (2020). Estilos de aprendizaje y ambiente de aula: situaciones que anteceden a la innovación pedagógica en estudiantes de deporte Learning style and student satisfaction: situations preceding the pedagogical innovation in a sports classroom. Retos: nuevas tendencias en educación física, deporte y recreación, 38(38), 213-221.

Cea, $M^{a}$.A. (2004), Métodos de encuesta. Madrid: Síntesis.

Creech, A., Gaunt, H., Hallam, S., \& Robertson, L. (2009), «Conservatoire students" perceptions of master classes». British Journal of Music Education, vol. 26, núm. 3,pp.315-331.

Conde, C. \& Almagro, B. (2013), «Estrategias para desarrollar la inteligencia emocional y la motivación en el alumnado de Educación Física». E-motion: Revista de Educación, Motricidad e Investigación, vol. 1, pp. 212-220.

De las Heras-Fernández, R. (2017), Propuesta de adaptación de métodos didácticos para el aprendizaje de conceptos rítmicos básicos aplicados a la enseñanza en el zapateado flamenco. el encaje silábico y la improvisación. Revista del Centro de Investigación Flamenco Telethusa, 10 (12) pp.11-18 http://www.flamencoinvestigacion.es/articulos1012-02-2017-propuestas-ritmo-flamenco/

Delgado, M.A. (1991). Los estilos de enseñanza en la Educación Física. I.C.E.: Universidad de Granada.

Espada, M., Fernández, M., \& Calero, J. C. (2019). «Validación de la versión española del Cuestionario de uso y percepción del espectro de estilos de enseñanza en educación física». Revista mexicana de investigación educativa, vol. 24, núm. 80,pp. 271-285. Disponible en http://www.scielo.org.mx/ $\mathrm{s}$ c i e 1 o . p p p ? p i d = S 14405 66662019000100271\&script=sci_arttext

Fernández, M. \& Espada, M. (2017). Formación inicial y percep- 
ción del profesorado sobre los estilos de enseñanza en Educación Física. Retos: nuevas tendencias en educación física, deporte y recreación, (31), 69-75.

Ferreiro-Pérez, A. (2015). Calidades de los vínculos educativos en la formación dancística profesional. Revista iberoamericana de educación superior, vol. 6, núm. 15, pp. 108-128.

Gamboa, J. M. \& Núñez, F. (2017), Diccionario de flamenco de la A a laZ. Diccionario de términos del flamenco, Madrid: Espasa Calpe, S.A.

Gérard, C. (1991). «El aprendizaje del ritmo musical». Comunicación, lenguaje y educación, vol. 3, núm. 10,pp.85-100.

Hemsy de Gainza, V. (2002), Música: amor y conflicto. Diez estudios de psicopedagogía musical. Buenos Aires: Lumen Humanitas.

Heras Monastero, B. D. L. (2013), «Acercamiento al estudio del baile flamenco desde el ámbito de la educación no formal». Encuentros con el PIE. Sevilla: Plataforma Independiente de Estudios Flamencos Modernos y Contemporáneos (PIE. FMC).

Jacquier, M. D. L. P., \& Pereira, A. (2010), «El rol del cuerpo en el aprendizaje del lenguaje musical. Reflexiones acerca del aporte de la cognición corporeizada». Actas del Seminario» Adquisición y Desarrollo del Lenguaje Musical en la Enseñanza Formal de la Música, pp. 67-72.

Jefatura del Estado (2006), Ley Orgánica 2/2006, de 3 de mayo, de Educación. BOE (04/05/2006), núm. 106, BOE-A-20067899 pp.45-47.

Junco, I. (2010), «La motivación en el proceso enseñanza-aprendizaje». Revista digital para profesionales de la enseñanza, vol. 9, pp. 1-14.

Juntunen, M. L., \& Hyvönen, L. (2004), «Embodiment in musical knowing: how body movement facilitates learning within Dalcroze Eurhythmics». British Journal of Music Education, vol. 21, núm. 2, pp. 199-214.

Kvale, S. (2011), Las entrevistas en investigación cualitativa. Madrid: Ediciones Morata.

Krathwohl, D.R. (2002), «A revisión of Bloom`s taxonomy: An overview». Theory into Practice vol. 41, pp. 212-18

Lago, P. (2006), «Música y creatividad, algo más que un lenguaje de expresión y de comunicación». Revista Prodiemus, vol. 2, pp. 1-12.

Ministerio de Educación y Ciencia. (2007), Real Decreto 85/ 2007, de 26 de enero, por el que se fijan los aspectos baisicos del curriìculo de las ensenPanzas profesionales de danza reguladas por la Ley Orgaìnica 2/2006, de 3 de mayo, de Educacioìn. BOE (13/02/2007), núm. 38, 2956, pp. 6249 6262

Ministerio de Educación. (2010), Real Decreto 632/2010, de 14 de mayo, por el que se regula el contenido básico de las enseñanzas artísticas superiores de Grado en Danza establecidas en la Ley Orgánica 2/2006, de 3 de mayo, de Educación. BOE(5/06/2010), núm.137,8956,pp. 48501 - 48516

Miquel, S., Bigné, E., Lévy, J.P., Cuenca, A.C. \& Miquel, Ma . J. (2000), Investigación de mercados. Madrid: Mc Graw-Hill.

Mosston, M., \& Ashworth, S. (1993), La Reforma de los Estilos de Enseñanza. Barcelona (España): Editorial Hispano Europea, S.A.

Mosston, M \& Ashworth, S. (2001), La enseñanza de la educación física. La reforma de los estilos de enseñanza. Barcelona, España: Hispano Europea.
Mosston, M. \& Ashworth, S. (2008), Teaching Physical Education, Nueva York: Pearson Education.

Nishida, T. (1984), «The effect of achievement motivation on motor skill learning». Japanese Journal of Physical Education, vol. 29, pp. 328-346.

Nishida, T. (1988), «Reliability and factor structure of the achievement motivation in physical education test». Journal of Sport and Exercise Psychology, vol. 10, pp. 418- 430.

Nishida, T. (1989), «Astudy on standardization of the achievement motivation in physical education test». Japan Journal of Physical Education, vol. 34, pp. 45-65.

Nishida, T. (1991), «Achievement motivation for learning in physical education class: A cross cultural study in four countries». Perceptual and Motor Skills, vol. 72, pp. 11831186.

Paramo, P. (2018), La Investigación en Ciencias Sociales: Técnicas de recolección de la información. Colombia: Universidad Piloto de Colombia.

Peñalver, J. M. (2013), «Análisis de la práctica de la improvisación musical en las distintas metodologías: características y criterios de clasificación». Artseduca, 4 pp. 74-85

Peñalver, J.M. (2019): «El lenguaje y la improvisación en el jazz.Construyendo un marco teórico para la educación musical (I). Objetivos y contenidos» En Revista Sonograma Magazine $\mathrm{n}^{\circ} 43$.

Ríos-Ruíz, M. (2002), El gran libro del Flamenco. Volumen 11. Intérpretes. Barcelona: Calambur.

Robledo Barros, R. (2003). Creatividad y cognición musical, procedimientos involucrados para el desempeño musical improvisativo. Cuadernos Interamericanos de investigación en Educación Musical, 3(005).

Rusinek, G. (2004). Aprendizaje musical significativo. Revista electrónica complutense de investigación en educación musical, 1, 1-16.

Ryan, R. M. \& Deci, E. L. (2000), «Self-determination theory and the facilitation of intrinsic motivation, social development, and well-being. The American Psychologist, vol. 55, núm. 1, 68-78.

Ruiz-Pérez, L. M., Moreno-Murcia J.A., Ramón-Otero, I \& AliasGarcía, A. (2015), «Motivación de Logro para Aprender en Educación Física: adaptación de la versión española del Test AMPET». Revista española de pedagogía, pp. 157-175.

Scribano, A., \& Sena, A. D. (2009). Las segundas partes sí pueden ser mejores: algunas reflexiones sobre el uso de datos secundarios en la investigación cualitativa. Sociologias, (22), 100-118.

Syrmpas, I. \& Digelidis, N. (2014), «Physical education student teachers' experiences with and perceptions of teaching styles»». Journal of Physical Education and Sport, vol. 14, núm. 1,pp. 52-59. doi: 10.7752/jpes.2014.01009

Torres, C. P., Ortega-Ruz, R., \& Hidalgo, A. J. R. (2017), «Integración de la especialidad de flamenco en el grado superior de música». Revista Electrónica Complutense de Investigación en Educación Musical, vol. 14, pp.351-368

Varguillas, C. (2006), «El uso de ATLAS. ti y la creatividad del investigador en el análisis cualitativo de contenido UPEL. Instituto Pedagógico Rural El Mácaro». Laurus, vol. 12, pp.73-87.

Wright, R. \& Kanellopoulos, P. (2010), «Informal music learning, improvisation and teacher education». British Journal of Music Education, vol. 27, núm. 1, pp. 71-87. 